\title{
ANGULAR NONRADIAL VS. USUAL RADIAL POTENTIAL ENERGY QUOTIENT
}

\author{
Jakub Czajko \\ Science/Mathematics Education Department \\ Southern University and A\&M College, Baton Rouge, LA 70813, USA \\ E-mail address: sunswing77@netscape.net
}

Keywords: Angular nonradial potential energy, potential energy quotient.

\begin{abstract}
Potential energy quotient of the angular nonradial to the usual radial potential energy (and the quotient's density for a closed orbit or an openended trajectory of a satellite) is offered without direct dependence on the mass of the given (assumed as nonrotating) celestial body (such as star or planet) but with explicit dependence on the body's density of matter.
\end{abstract}

\section{INTRODUCTION}

Even nonmathematician can see that if the operationally correct (and thus mathematically quite legitimate) product differentiation rule (PDR), which is written here in terms relevant to radial force fields, namely of the vector of the gravitational force $\mathbf{F}$ and the distance pointing vector $\mathbf{r}$ :

$$
\mathrm{dW}(\mathbf{F} \cdot \mathbf{r}):=\mathrm{d}(\mathbf{F} \bullet \mathbf{r})=\mathbf{F} \cdot \mathrm{d} \mathbf{r}+\mathbf{r} \cdot \mathrm{d} \mathbf{F}
$$

which is proven true and thus is absolutely mandatory to be always applied for any products, then the traditional definition of work done rate (meant as the scalar product of the force vector $\mathbf{F}$ over the straightlinear distance $\mathrm{r}=|\mathbf{r}|$ to the given trajectory/orbit pointed by the distance pointing vector $\mathbf{r}$ yet mistakenly designated as product of force over distance change rate $\mathrm{d} \mathbf{r}$ ):

$$
\mathrm{dW}:=\mathbf{F} \cdot \mathrm{d} \mathbf{r}
$$

is incomplete, and so is also the potential energy that is spent on the work done by the given force field. Yet the operationally wrong and conceptually faulty formula (2) is used in all textbooks and lectures [1] despite the (proven) fact that - according to Frenet-Serret (FS) formulas of differential geometry - the formerly omitted nonradial term $\mathbf{r} \bullet \mathbf{d F}$ (highlighted there in red) is unavoidable in general [2]. The substitution (2) is a formula for mathematical disaster. If it had been defined with correct mathematical insight as: $\mathrm{dW}:=\mathrm{d}(\mathbf{F} \bullet \mathbf{r})$, as it is interpreted, it would lead to the right equation (1).

Although making mistakes is natural and sometimes could even be beneficial [3], tacitly concealing them and then covering them up with fake theorems [1] misleads some students and researchers with lesser mathematical acumen, which can amplify the adverse effects of the original mistake on understanding of the infected topics.

According to the PDR, operationally valid rate of work done should be evaluated [4] as:

$$
\begin{gathered}
\mathrm{dW}(\mathbf{F} \cdot \mathbf{r})=-\mathrm{F} \cos 2 \alpha \mathrm{dr}+2 \mathrm{Fr} \sin 2 \alpha \mathrm{d} \alpha-\mathrm{r} \cos 2 \alpha \mathrm{dF} \\
=-\mathrm{dW}(\mathrm{r})+\mathrm{dW}(\alpha)-\mathrm{dW}(\mathrm{F})
\end{gathered}
$$

where $\alpha$ is the angle of visibility of trajectory/orbit of a satellite (or that of an electron in an atom in the Bohr's picture) as seen from the local gravity center when sweeping its perihelion radius.

The formula (3) contains the usual radial-only work done rate $\mathrm{dW}(\mathrm{r})$ as well as the two formerly disregarded components of work done, namely the angular nonradial rate $\mathrm{dW}(\alpha)$ and the linear nonradial rate $\mathrm{dW}(\mathrm{F})$ of the total work done. Note that the rates of work done pertain also to 
rates of the field's potential energy that is spent on the work done by the given radial/center-bound force field. Although in this presentation the force field is gravitational, it could be of electrostatic origin too. If treated as stationary, these fields are conceptually and mathematically similar.

Notice that sign of the angular nonradial term is opposite to the signs of the attractive terms the usual radial and the linear nonradial term. This fact gives rise to - formerly unknown - angular repulsive force of the usual radial gravitational field in which it is intrinsically embedded.

Since no law of physics is complete but depends on the depth of inquiry into the phenomena under investigation, I shall specify the particular level/depth of inquiry used in this presentation. At the present depth of inquiry, I will consider only gravitational interactions happening within the usual stationary radial/center-bound force fields of nonrotating celestial bodies with fixed masses and assumed as constant average densities of matter of the source masses that generate the fields.

The more advanced is mathematical representation of a physical phenomenon the larger is its conceptual scope and the higher is also the total number of variables and parameters involved in its more nuanced description. This assertion is supported by mathematics, which evidently allows for enhancements via discovery of previously unrecognized (hence hidden) variables [5]. In a sense thus, the dream of a simple and elegant single final equation with minimal number of variables is just that: a pipe dream. While on the subject, notice that just two variables working in tandem must produce four components. The eq. (3) has three independently varying magnitudes only because the angle of visibility is the same for both: the force vector and the pointing radius vector.

The ultimate goal of scientific investigations should be truth, not elegance or perhaps some other artistic qualities of prospective scientific laws. Such objectives as simplicity of representation or the minimal number of variables are certainly desirable, but they must not replace the goal. The eq. (3) suggests superposition of the radial and nonradial components of interactions taking place within radial/center-bound force fields. Whether one likes it or not, the eq. (3) is a mathematical prescription for the physical interactions, which has already been confirmed in experiments [6].

To the detractors who pretend that the faulty eq. (2) is an approximation I should say that it is not. If the eq. would be derived from the eq. (1) then it could be considered as its approximation, but in that case one would have to justify the reason for the approximation and perhaps estimate its error. With notable exception of Mercier who questioned the validity of the former representation of potential energy [7], [8], and few other researchers, the mystification of potential energy continues even today, despite all the - now explained - rebutting experimental evidence [6], [9].

It is quite possible that between AD 1773 (since Lagrange realized that force fields seem to be endowed with what is called today potential [10]) and AD 2000, when it has been shown that nonradial effects of gravity do exist indeed [6], someone was trying to portrait the faulty eq. (2) as approximating the PDR, but solutions of the differential equations (1) and (3) did not materialize until recently [1]. As a matter of fact, it is possible to enhance the eq. (3) even further [5].

\section{SUPERPOSING ANGULAR NONRADIAL AND RADIAL POTENTIAL ENERGY}

When substituting the magnitude of the usual radial force vector of classical gravitational force field:

$$
\mathrm{F}=|\mathbf{F}|=\frac{-\mathrm{GMm}}{\mathrm{r}^{2}}
$$

into the differential equation (3) and then solve it with respect to the radial distance $r$ alone, we obtain

$$
\mathrm{W}(\mathrm{r})=\frac{\mathrm{GMm}}{\mathrm{r}}
$$


which is the usual (i.e. radial-only) amount (i.e. functional) of potential energy at the perihelion of the trajectory/orbit of the mass $m$ (i.e. $\alpha=0$ ) that is passing by (or orbiting) the (assumed as big) source mass $\mathrm{M}$ that generates the locally dominant gravitational field. $\mathrm{G}$ is the gravitational constant.

Solving the equation (3) with respect to the originally planar angle (of visibility of the orbit or trajectory) $\alpha$ alone, which during motion of the mass $\mathrm{m}$ turns into twisting spherical/spheroidal angle $\theta$ (of the angular distance $\lambda=r \theta$ that is measured along an equipotential surface) we get in general:

$$
W(\alpha)=W(Q(\lambda))=\frac{-4 \mathrm{kGMmr} r_{\mathrm{p}}^{2} \lambda}{3 \mathrm{Q} \mathrm{r}^{4}}=\frac{-\mathrm{kuGMm} \lambda}{\pi \mathrm{r}_{\mathrm{p}} \mathrm{Qr}}
$$

which is the angular nonradial amount of potential energy spent on the work done by the field along the equipotential surface at the perihelion distance $r_{p}$ that is also codetermined by the radial distance $r$, and the (assumed as average - at the present, simplified depth of inquiry into the given gravitational phenomena) density of matter $\mathrm{Q}$ of the source mass $\mathrm{M}$, and by the volume $v$ enclosed within the perihelion [11], [9]. The value of proportionality constant $\mathrm{k}$ proved (by experiments) to be 1 for practically spherical celestial bodies such as our Sun and Earth [6], near their respective surfaces. It is also needed for equalizing units (such as radians or angular degrees).

The constant density of matter functional Q, which is practically fixed parameter for planets, is nevertheless also a function of exposure. Although fixed function of exposure does not change its own value, it allows accumulation of the (nonradial) impact it exerts on the orbiting small test mass $\mathrm{m}$ along the angular distance or during the time of its exposure to the big mass M. It is analogous to exposure to heat or radiation.

On the first page of his Principia Newton defined quantity of matter, which he called mass, as magnitude arising from both: density and bulk of material substance ascribed to every massive body, whereas quantity of motion should arise from velocity of the body and its mass combined [12]. Newton was correct in his insistence that density of matter should have an impact on some aspects of gravitational interactions, but he did not identify them, and thus his gravitational mass became effectively synonymous with the bulk of matter. It was one of two main problems - the other being the fact that without gravitational repulsion the universe should have collapsed - that presumably caused his hesitation, which postponed the publication of Principia by some 20 years.

The eq. (6) vindicates the Newton's suspicion that density of matter does indeed influence (nonradial) gravitational interactions. The fact that the angular nonradial effect has opposite sign to the usual radial one - as indicated in both the eq. (3) as well as by comparison between the eq. (5) and (6) - means that the universe is not in danger of collapse because the angular nonradial component gives rise to gravitational repulsion intrinsic to all gravitational interactions in general.

The actual physical existence of the nonradial (i.e. tangential and/or binormal) effect of radial gravitational fields, which means exposure to the mass source of the locally dominant gravitational field, has already been confirmed in two unbiased direct experiments as well as indirectly in several other experiments and observations [6].

Note that exposure to the impact of mass was theoretically unknown in mainstream physics due to the faulty formula (2), although it was occasionally speculated about. Nonetheless, it was well known to practitioners that seas, oceans and landmasses adversely affect propagation of radio signals of the land-based differential positioning system that was used for navigation of sea-faring ships in the past (see select references in [6] that attest to presence of the latter effect). It should be conceptually rather self-evident, that if nominally massless photons and radio waves can feel some clearly nonradial impact of the celestial bodies that happen to be near their trajectories, then other massive bodies should also be affected by the celestial bodies. We should devise means to measure that effect in terms pertaining to the trajectory/orbit whereon the interaction occurs. 


\section{QUOTIENT OF THE ANGULAR NONRADIAL TO THE USUAL RADIAL POTENTIAL ENERGY}

Let us define the quotient $\mathrm{q}$ of the angular nonradial potential energy to the usual radial potential energy as:

$$
\mathrm{q}:=\frac{\mathrm{w}(\mathrm{Q}(\lambda))}{\mathrm{w}(\mathrm{r})}=\frac{-\mathrm{kv} \lambda}{\pi \mathrm{Qr} \mathrm{r}^{\mathrm{r}} \mathrm{s}^{\mathrm{s}}}=\frac{-\mathrm{kv} \theta}{\mathrm{AQr} \mathrm{p}}
$$

which is written in terms of perimeter volume $v\left(r_{p}\right)=4 \pi\left(r_{p}\right)^{3} / 3$ and the area $A(r)=\pi r^{2}$ determined at a point that is pointed to the radius $r$ of generic orbit. Notice that the area does not refer to the orbit itself, which is an ellipse if planar, but to the circular disk that is attached to every generic moving point of the trajectory taken by the satellite of mass $m$ (or to its orbit in particular).

Note that $v$ is the perimeter volume enclosed by the fixed distance $r_{p}$ to the perihelion from the local gravity center of the mass M. Since the equipotential distance $\lambda=r \theta$ is codetermined also by the radial distance $r$ (which is limited from below by the radius of the perihelion $\mathbf{r}_{\mathrm{p}}$ ) and by the spherical angle $\theta$ (which can be curvilinear, i.e. spheroidal spherical in general) that corresponds to the angular equipotential path $\lambda$, the area $A=\pi r^{2}$ pertains to an idealized planar disk of the generic orbit around the celestial body.

In a sense thus, the quotient could be used to chart distribution of the ratio of the angular nonradial to the usual radial effects of gravity. Since it has no direct dependence on the mass of the celestial body (and thus also on its motion), the quotient could be used to distinguish the radial gravitational redshift proposed by Einstein' general theory of relativity (GTR) that does indicate distance to the star from its nonradial gravitational redshift, which is due to proximity of the line of sight of the light coming from the star to other stars or planets nearby. The new angular nonradial redshift [6] may also help to explain the extraneous redshifts of some quasars, the distances to which seem to place them far beyond the galaxies they apparently belong to [13], and that the universe appears to be younger than some objects within it [14], [15].

The common argument that redshift of quasars indicates their distance relied on the fact that brightness and redshift of quasars were related [16].

Hoyle admitted the possibility that redshifts of some quasars may not be related to distances [17] and some apparently distant quasars may be not that far away [18]. Not all redshifts are due to expansion [19]. Besides the three (classical) possible sources for the redshifts of astronomical objects (namely: Doppler effect, cosmological redshift and the [radial] gravitational redshift [20]), the electromagnetic redshift [21] and the new, distinct relativistic redshift that was found theoretically [22], the angular nonradial effect causes nonradial gravitational redshift. Actually, it causes two formally independent redshifts: the tangential and binormal one [6] that complement the usual radial redshift predicted by the GTR.

Note that the angular nonradial effect of radial gravity is due to the fact, that the light from a distant star (or the orbiting mass $\mathrm{m}$ ) goes around the source mass M [6]. Therefore most distances to distant stars estimated via the radial gravitational frequency shift may be overestimated, because the nonradial effect of radial gravitational fields was disregarded due to blind adherence to the faulty formula (2). The two - formerly unexplained, due to blind acceptance of the faulty formula (2) experiments conducted by Sadeh [23], [24], clearly showed the nonradial effects of our Sun and the Earth, respectively. The experiments have been explained via nonradial effects of radial gravity in [6] and they are discussed in [9] too. It was noted that no previous (i.e. devised before 2000 AD) gravitational or electromagnetic theory of physics could explain the formerly quite unanticipated frequency decrease found in the two experiments [25].

The theory of nonradial effects of gravity does not invalidate the GTR, but complements it, because the GTR is valid only for purely radial phenomena. Einstein admitted that in [26]. Like most other scientists, his thinking was influenced by the faulty formula (2). The price he paid for that was inability to make exact determination of his flagship prediction of deflection of light by 
Sun. Since the deflection is not entirely radial but partly tangential (i.e. nonradial) phenomenon, the nonradial effect of gravity corrected his inaccurate prediction in accordance with the results of most precise experimental data [6]. The GTR is incomplete and has problem with localization of energy within its amalgamated gravitational field - the latter aspect shall be discussed elsewhere.

\section{DENSITY OF THE POTENTIAL ENERGY QUOTIENT}

Given the formal independence of the perimeter volume and the twisted area that is loosely associated with an idealized orbit, which could nevertheless be assigned to each point moving along the orbit/trajectory, one may define the density $\chi$ of the potential energy quotient as follows:

$$
\chi:=\frac{\mathrm{q}}{\mathrm{v}}=\frac{-\mathrm{k} \lambda}{\pi \mathrm{Q} \mathrm{r}_{\mathrm{p}} \mathrm{r}^{\mathrm{s}}}=\frac{-\mathrm{k} \theta}{\mathrm{AQ} \mathrm{r}_{\mathrm{p}}}
$$

which can relate the density of matter functional $[\mathrm{Q}(\lambda)]$ to the twisting trajectory/orbit in either the terms of the angular distance $\lambda$, or as the (alternative) functional $[\mathrm{Q}(\theta)]$ represented in terms of the spherical/spheroidal angle (that is associated with the angular distance).

Although the quotient and its density are represented here as scalar measures, the repulsive angular nonradial potential that causes twisting of trajectories is orthogonal to the usual attractive radial potential and therefore the repulsion does not scalarly diminish the attractive radial force, but rather redirects it sidewise. In the sense the angular nonradial potential has also dissipative effect in addition to its repulsive action causing the twisting. This aspect shall be discussed elsewhere.

The eq. (8) clearly indicates that density of the mass-independent quotient of the potential energy that is involved in field interactions has diminishing but inversely proportional relation to all the predictors that codetermine it: The twisting area A, the density of matter of the source mass $\mathrm{M}$ and the perihelion radius of the trajectory/orbit along which the satellite (mass $\mathrm{m}$ ) moves.

\section{CONCLUSION}

Since the angular nonradial and the usual radial components of potential energy involved in gravitational interactions within radial/center-bound force fields have opposite signs, they give rise to repulsive and attractive force fields, respectively. Therefore, determination of the combined functional of potential energy of the interactions can use the mass-independent quotient of their contributions as well as density of the quotient.

Since gravitational interactions take place along the open trajectory or closed orbit on which the satellite moves, the quotient and its density circumvent the need to know exactly all the mass sources that contribute to the locally dominant field.

Unlike masses, which are directly proportional to the potential energy, all the functionals that codetermine the quotient and its density are (formally) inversely proportional to these measures, whereas the independently varying variables involved therein (either the angular distance or the spherical/spheroidal twisting angle that corresponds to the angular distance) are always directly proportional to these two measures.

\section{References}

[1] Czajko J. Path-independence of work done theorem is invalid in center-bound force fields. Stud. Math. Sci. 7(2) (2013) 25-39 http://cscanada.net/index.php/sms/article/view/j.sms.1923845220130702.2469/5279 .

[2] Czajko J. Radial and nonradial effects in Frenet frame. Appl. Phys. Res. 3(1) (2011) 2-7 http://www.ccsenet.org/journal/index.php/apr/article/viewFile/9555/7421 .

[3] Ohanian H.C. Einstein's mistakes. The human failings of a genius. New York: W.W. Norton, 2008, p.XIX.

[4] Czajko J. Equipotential energy exchange depends on density of matter. Stud. Math. Sci. 7(2) (2013) 40-54

http://cscanada.net/index.php/sms/article/view/j.sms.1923845220130702.2752/5336. 
[5] Czajko J. Mathematical gateway to complementary hidden variables in macrophysics. International Letters of Chemistry, Physics and Astronomy 50 (2015) 117-142 http://www.scipress.com/ILCPA.50 May 2015.

[6] Czajko J, On conjugate complex time II: Equipotential effect of gravity retrodicts differential and predicts apparent anomalous rotation of the Sun. Chaos, Solit. Fract. 11 (2000) 2001-2016.

[7] Mercier A. Analytical and canonical formalism in physics. Amsterdam: North-Holland, 1959, p.122.

[8] Mercier A. Speculative remarks on physics in general and relativity in particular. [pp.295-303 in: De Sabbata, V. \& Weber, J. (Eds.) Topics in theoretical and experimental gravitation physics. London: Plenum Press, 1977].

[9] Czajko J. Galilei was wrong: Angular nonradial effects of radial gravity depend on density of matter. International Letters of Chemistry, Physics and Astronomy 11(2) (2014) 89-105 http://www.ilcpa.pl/wp-content/uploads/2013/10/ILCPA-112-2014-89-105.pdf.

[10] Birkhoff G. (Ed.) A source book in classical analysis. Cambridge, MA: Harvard Univ. Press, 1973, pp.335, 360.

[11] Czajko J. Operationally complete work done suggests presence of potentials corresponding to repulsive forces. International Letters of Chemistry, Physics and Astronomy 18 (2014) 16-30 http://www.ilcpa.pl/wp-content/uploads/2013/10/ILCPA-18-2014-16-30.pdf.

[12] Newton I. Principia. Edited and commented by Stephen Hawking. Philadelphia: Running Press, 2002, p.1.

[13] Shipman H.L. Black holes, quasars, and the universe. Boston: Houghton Mifflin, 1980, p.239.

[14] Davies P.C.W. Einstein's greatest mistake? Astrophys. Space Sci. 244 (1996) 219-227.

[15] Kellermann K.I. Superluminal radio sources. Comments Astrophys. 11C (1985) 69.

[16] Pfleiderer J. Are quasar redshifts distance-dependent? ZS. Astroph. 69 (1968) 140-145.

[17] Hoyle F. Review of recent developments in cosmology. Proc. R. Soc. Lond. A308 (1968) 1.

[18] Hodge P.W. Concepts of the universe. New York: McGraw-Hill, 1969, p.110.

[19] Burbidge G. Two universes. Astrophys. Space Sci. 244 (1996) 169-176.

[20] Kaufmann W.J., III. Relativity and cosmology. New York: Harper \& Row, 1973.

[21] Apsel D. Time dilations in bound muon decay. GRG 13 (1981) 605-607.

[22] Ihrig E. A local relativistic red-shift effect. GRG 8 (1977) 877-885.

[23] Sadeh D., Knowles S.H. \& Yaplee B.S. Search for a frequency shift of the 21-centimeter line from Taurus A near occultation by Sun. Science 159 (1968) 307-308.

[24] Sadeh D., Knowles S. \& Au B. The effect of mass on frequency. Science 161 (1968) 567-569.

[25] Szekeres G. Effect of gravitation on frequency. Nature 220 (1968) 1116-8.

[26] Einstein A. The Foundations of the General Theory of Relativity. [pp.111-164 in: H.A. Lorentz et al. The principle of relativity. New York: Dover, 1923, see p.161]. 